\title{
Unifying conceptual and spatial relationships between objects in HCI
}

\author{
David Blezinger (1), Ava Fatah gen. Schieck (1), Christoph Hölscher (2) \\ (1) Bartlett School of Graduate Studies \\ Central House, 14 Upper Woburn Place, London WC1H 0NN, UK \\ (2) Center for Cognitive Science, Institute for Computer Science and Social Research \\ Friedrichstr. 50, 79098 Freiburg, Germany
}

\begin{abstract}
To design interfaces which occupy a continuous space of interaction, the conceptual model of an interface needs to be transferred to a spatial model. To find mappings between conceptual and spatial structure which are natural to people, an experiment is undertaken in which participants organize objects in a semi-circle of shelves around their body. It is analyzed how conceptual relationships between objects such as categorial relationships and sequential relationships within task performance are represented in spatial configurations of objects as chosen by the participants. In these configurations, a strong correlation between conceptual and spatial relationships is observed between objects.
\end{abstract}

Keywords: HCI frameworks, spatial interface, conceptual model, information architecture, navigation, object-based, task-based, spatial configuration, spatial cognition, embodied interaction, categories, visual identity

\section{Introduction}

Before the information architecture of a Human-Computer Interface is implemented in wireframes and visual designs, conceptual models are used to develop relationships between objects [1][2][3]. Within conceptual maps, objects are spatially related to each other, but this spatiality is not transferred to the visual layout of the final interface.

Although the necessity to incorporate space into interface design is emphasized in the literature [4][5] there is currently no framework that allows a mapping of conceptual relationships between objects to spatial relationships between the same objects. Instead, objects are laid out in consecutive windows which are not spatially related to each other. This divides the space through which the user navigates rather than providing a continuous space that she encounters in her natural, non-digital environment.

Interaction within a continuous space is not restricted to a certain form of interface. It can equally be applied to screen interfaces, tangible interfaces and other forms of spatial interfaces. More important than the technical or material features of the inter-

adfa, p. 1, 2011.

(C) Springer-Verlag Berlin Heidelberg 2011 
face is the way in which its objects relate to each other. Although a spatial interface can be an interface that takes up 3D space around the human body, it can also be a screen based interface at smaller scale in which objects of use are related to each other in one continuous visual space.

To inform a layout of objects in a spatial interface it needs to be known what types of relationships between these objects are relevant to the user's perception, cognition and interaction, and how these relationships can be mapped to spatial relationships between the same objects.

We take categorial relationships between objects and relationships of sequential order of objects within task performance as two main factors which determine the concepualization of object relationships in people's cognition (potentially related to [6][7]), and which are likely to strongly influence the way in which participants of our experiment will choose to organize objects spatially.

We use a conception of tasks that include only such tasks which consist of movement between objects as it would occur in navigating a window-based interface, but in a continuous space. We exclude tasks that involve the manipulation of objects, to create a more uniform experimental setup.

Both categorial relationships between objects and relationships of sequence in which objects follow each other within task performance exist independently of the spatial layout in which objects are laid out and tasks are performed. Thus, these relationships can be treated as conceptual relationships between objects that can be designed within a conceptual model before implementing the interface spatially (for category effects on spatial search see [8]). To enable such an implementation, principles by which conceptual relationships between objects can be mapped to spatial relationships between the same objects need to be found.

By spatial relationships, we mean relationships between objects as relevant to individual perception, cognition and interaction (for embodied interaction with objects see [9][10]). Thus, our concept of spatial relationships is tied not only to relationships between objects but also to the relationship between the human body and these objects. Thus, spatial relationships between objects as described here are relationships as perceived and conceptualized in cognition [11], and are thus tied to the viewpoint of the perceiving, thinking and interacting individual.

We represent the human body through its standing point and reach of arms, which leads to a sphere of possible movement trajectories around the human body [12]. Tasks that include navigation towards several objects in a certain sequence can thus be represented as trajectories of movement between spatially distributed objects in relationship to the human body. In our experimental setup, we choose an angular distribution of objects around the participant's standing point to match this sphere of performative body movement, thus enabling to integrate the representation of body movement and the representation of the spatial environment in which the experiment is performed. This allows an analysis of the influence of movement, perception and cognition on object relationships within an environmental representation.

The spatial framework model [13] states that the position of objects in one's spatial environment are memorized in relationship to the axes of the human body. We aim to 
relate our experimental setup to the left/right axis and to the top/bottom axis, integrating standing point and movement of the participant with object related cognition.

\section{The Experiment}

The design of the experimental setup was inspired by an analysis of task performance within a kitchen environment. In a kitchen, objects of daily use are stored in cupboards and shelves. To perform a task such as making coffee, a set of objects is taken from their storage position to a working surface where the task is performed. Each time groceries are bought at the store, they need to be stored in the shelves and cupboards. The objects are stored in relationship to objects which are already present in space, and when no relationship can be found, a new location needs to be found, or the overall configuration of objects needs to be changed to accommodate the new objects.

The experimental setup is designed to contain both activities, and to potentially reveal a mutual influence between the two. First, the participant needs to organise a set of objects in shelves, as she would do it with objects of her daily life. Second, she performs a set of four tasks with these objects, and third, she organises the objects in the shelves again, after the experience of the tasks.

We adapted the environment and the way tasks are performed (Fig.1), to suit our method of analysis and to allow the findings to inform the design of HCI. As described above, the spatial environment in our experimental setup forms a near semicircle around the participant's standing point, thus equaling the distance at which the participant can position objects with her hands. By choosing this layout and by placing our shelves at a convenient height, we strive to minimize the influence of ergonomic factors on the spatial positions of objects that the participant chooses. Cognitive rather than ergonomic factors are in the focus of the study.

As mentioned above, we define tasks as sequences of object use and thus sequences of movement between objects. Instead of placing the objects on the

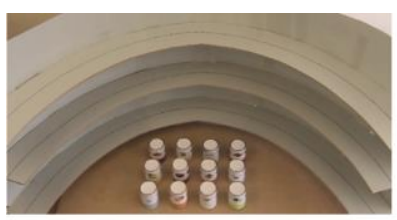

1- Initial positions of objects

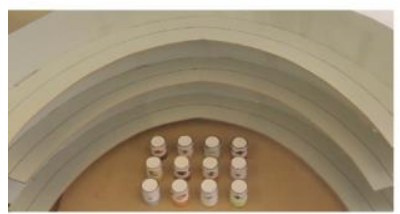

4 - Initial positions of objects

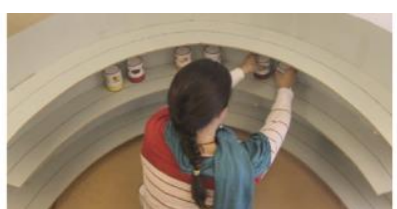

2 - Placing the objects in the shelves

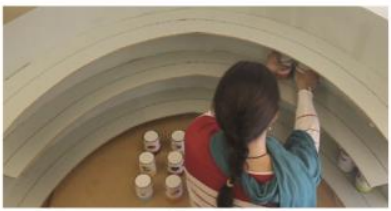

5 - Placing the objects again

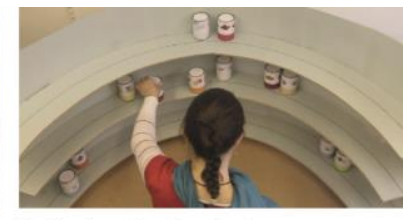

3-Performing the task sequences

Fig. 1. The order in which each participant performs the experiment 
working surface as it would occur in a kitchen environment, tasks in our experimental environment only require grabbing the objects at their spatial location within the shelves and moving them from the back to the front of the shelves. We assume that this action will reinforce the memory of the spatial position of an object. To further reinforce the memory of the spatial configuration of objects in relationship to task performance, the participant needs to perform the set of tasks twice.

The objects are chosen to form categorial relationships of variable strengths. Common categories (fruits, sweeteners, glasses) are chosen which are likely to be shared across participants, even from different cultural backgrounds. To put the focus on cognitive / spatial relationships between objects, the influence of affordances (shape, size) [14] on the organization of objects is eliminated by choosing an equal size, shape and weight for all objects. The graphic identity of each object consists of a picture of the visual appearance of the actual object that the uni-size object represents, and of a color band. In the first object set which we will call „match“, the color is chosen to match each object and thus its category (e.g. a red color is chosen for raspberries and a green color for limes).

We expect that the categorial relationships will be strongly represented in the spatial configuration participants choose when organizing the objects in relation to each other. Participants are likely to group objects of the same category together. We expect that the repeated performance of sequential movements (tasks) on a set of spatially distributed objects will have an influence on the conceptualization of relationships between objects and may thus lead to a re-configuration of objects when spatially organizing the objects again, after task performance.

To test whether the influence of color or categorial information of objects on their spatial organization is stronger, we introduce a second object set in which the color does not match the object. Instead, the pictures of the objects are displayed in greyscale, and the color is chosen randomly, not matching the true color of each object. We call this object set „no match“. The participants are divided into two groups. Group A performs the experiment with object set „,no match“, group B with object set „match“. Each group contains 16 participants, each of which takes about 20 minutes to perform the experiment.

\subsection{Analysis of object distributions}

To find a simple yet suitable computational and visual representation that enables an analysis of the results, we map the angular distribution of objects within the shelves onto a plane. This enables a maintenance of angular information while adapting the representation to a more versatile $2 \mathrm{D}$ plane. The preservation of metric information would be possible using this representation, but as spatial-configurational relationships between objects rather than metric information are at the focus of the experiment, a metrically correct mapping of the experimental setup was not required. 
Each object distribution within the shelves, as chosen by the participant, is transferred to an excel spreadsheet which is saved as a csv file for further computational analysis and visualisation (Fig. 2). Each column of the spreadsheet represents a part of the shelf which has the width of one object. There are three rows per layer of shelves, to represent the numerical identity of each object, and additional information about the grouping of objects.

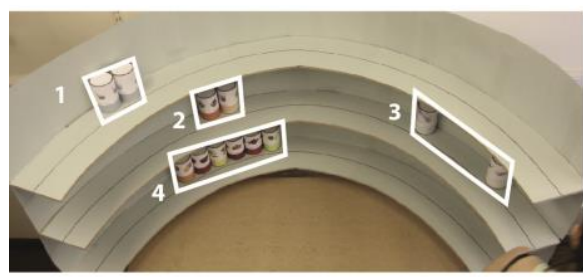

Object distribution and clustering

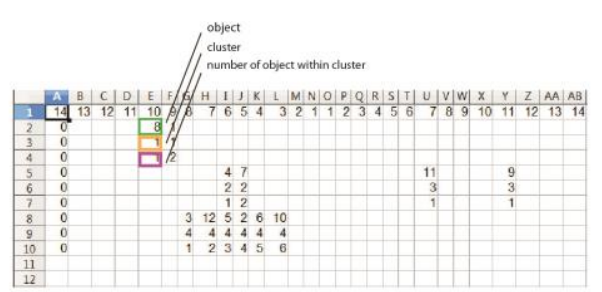

Computational representation of object distribution

Fig. 2. Transferring the spatial distribution of objects to a spreadsheet

Based on the data entered in the spreadsheet, the spatial distribution of objects and the sequential movement between the objects within the tasks are visualized (Fig.3).

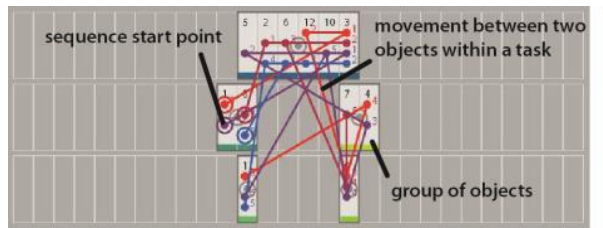

Visualisation of the trajectories of task sequences

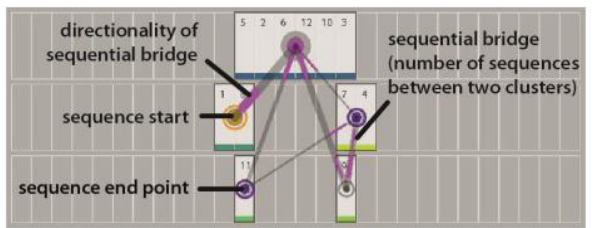

Visualisation of movement between clusters

Fig. 3. Visualisation of movement between objects and between groups of objects

\section{Results and Discussion}

In our analysis of the experimental findings, we focus on spatial relationships between objects and how these relate to the conceptual relationships between the same objects (categorial relationships and task-based, sequential relationships). The large variation in geometric patterns across participants shows that individual choice plays a large part in spatially organizing objects. However, it seems that there are factors which constrain the variation in pattern generation and lead to the observation of repetition in the rules by which the patterns are formed. Different types of spatial organisation can be differentiated by the size of object groups, by the extension at which the objects are distributed in the shelves, by the simplicity of sequential pathways between object groups and by the change of group size and group content. 


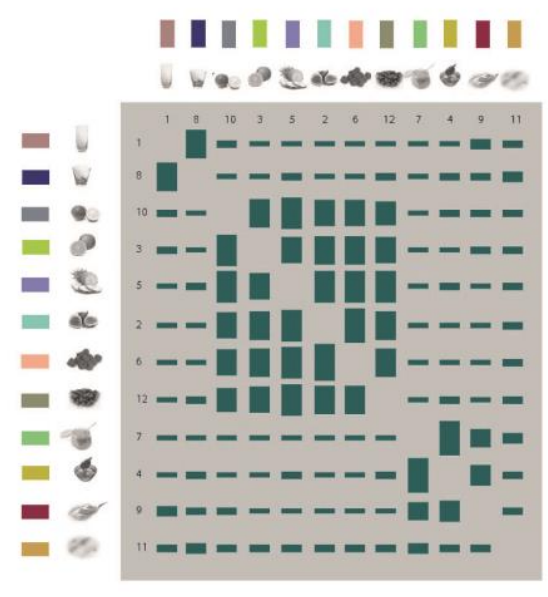

Group A

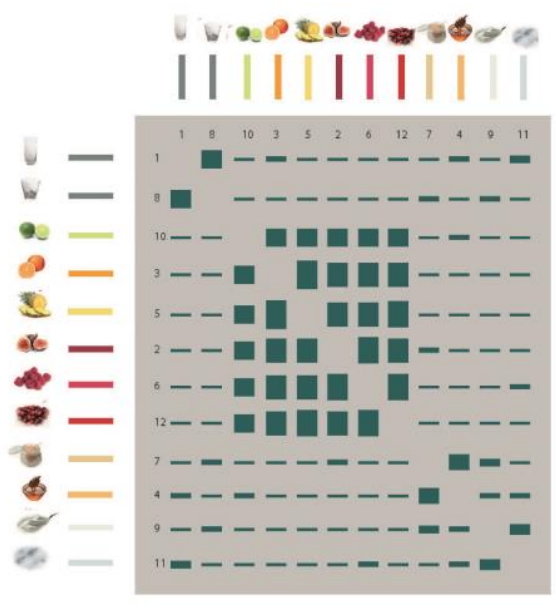

Group B

Fig. 4. Relative frequency of object pairs which remain grouped in both object distributions

\subsection{Grouping of objects}

When placing objects in shelves, participants tend to group objects together. In our spreadsheet, we gathered information about which objects are grouped together in each of the two object distributions. This enables an analysis of object grouping across all participants of each group. We analyse the frequency with which pairs of objects remain grouped together in both object distributions, and compare the results of both participant groups to each other. As described above, the colour of each object matches the categorial identity of the object in group B, while the colours are chosen randomly in group A.

In both participant groups, objects of the same category remain grouped most frequently (Fig.4), which indicates that categorial information has a strong influence on the grouping of objects while colour does not serve as a dominant principle of organising objects into groups. When categorial information is available, no influence of colour on the grouping of objects can be observed. However, within groups the objects were frequently organised in a colour gradient in both participant groups.

\subsection{Patterns of spatial positions}

In some cases, it appears that objects are organized by spatial positions and patterns among these spatial positions independent of the actual objects stored at these positions. It can be observed that some participants choose almost the same pattern between spatial positions at which groups of objects are stored when they place the objects for the second time while the objects themselves are exchanged between different spatial positions. (Fig.5a) Sometimes, symmetry is used to order objects into geometric patterns, and simple geometric patterns such as triangular or rectangular shapes between object positions can be frequently observed (Fig.5b). 


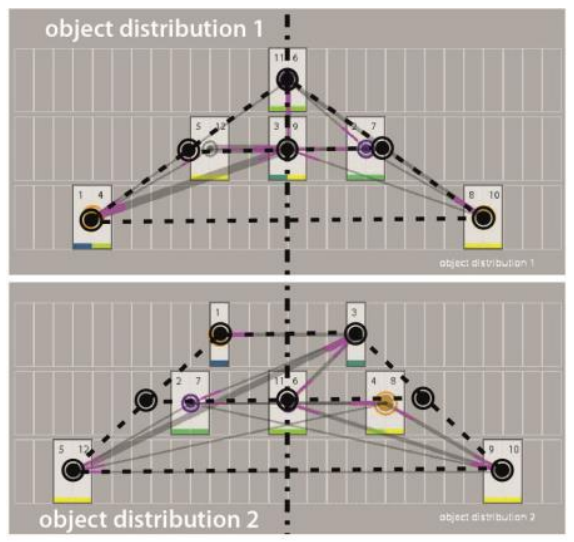

Fig. 5a: "Spread groups of objects"

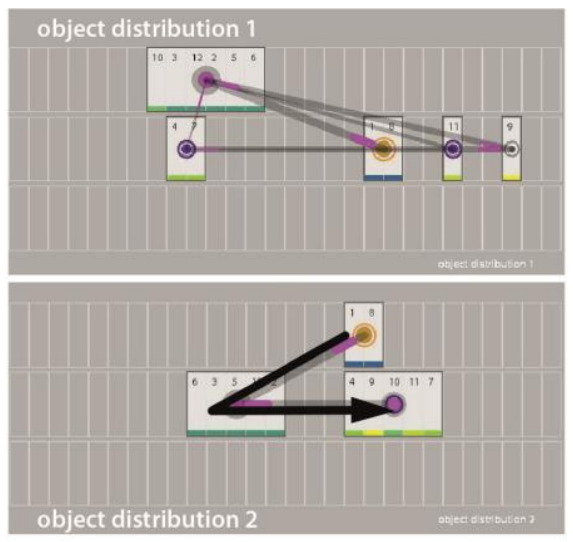

Fig. 5b: "Concentrated groups of objects"

Fig. 5. Examples of different types of spatial organisation

\section{3 organisation by sequences of movement}

By re-configuring objects and object groups in relationship to each other, the pathways that a set of movement (task) sequences take across a set of object groups can be simplified, creating a space of movement which is reduced to a low number of remaining pathways between object groups. In the second object distribution, the number of object groups is lower than in the first, and the number of movement pathways between groups is also reduced (Fig.5b). However, the influence of tasks and movement on the re-organisation of objects cannot be clearly defined, as the grouping of objects acts as a concurring or complementary organisational principle.

The results deliver a set of organisational principles that could be applied in the design of Human-Computer Interfaces. Because these principles are derived from an experiment in which participants were asked to organize objects as they would usually do it, the organizational principles derived from the experiment are likely to be natural to human perception, cognition and interaction.

Categorially related objects can be grouped together, colours can be used to organise objects in gradients, geometric patterns of spatial positions can serve to organise objects within an interface, and the space of task sequences can be simplified by re-configuring the objects.

The results suggest that geometric patterns of spatial positions of objects or groups of objects can serve as features to organize objects spatially and to give orientation in interaction, independently of the content that is stored at each spatial position. Thus, spatial positions can serve as ,containers" for objects, and patterns between them can facilitate the memorization of a spatial system of containers. Strong geometric patterns such as triangles between spatial positions or symmetrically ordered spatial positions appear repeatedly. This may be due to their property of facilitating orientation within the space of interaction. 
Giving an interface a spatial structure can be partly achieved by mapping conceptual relationships between objects to spatial relationships. However, some aspects of object organization, such as the formation of geometric patterns between spatial positions of objects and clusters have a purely spatial component which cannot be generated by a mapping from a conceptual model alone.

\section{Conclusion}

To design interfaces that occupy a continuous space of interaction, the conceptual model of an interface needs to be transferred to a spatial model. To find mappings between conceptual and spatial structure that are natural to people, an experiment was undertaken in which participants organise objects in a semi-circle of shelves around their body.

The experimental findings may provide principles which can inform a mapping from a conceptual model to a spatial interface structure and which are natural to human perception, cognition and interaction. Thus an implementation of the observed principles into the design of Human-Computer interfaces could potentially make an interaction more natural to the users.

In common window-based interfaces, the desktop is used by many people to store their most relevant files and folders. The findings of this study, supported by further research, could enable an implementation of organisational relationships between objects into the spatial and visual organisation within the desktop. Hierarchical and associative relationships between objects which are otherwise hidden in folder structures could be spatially and visually present within a continuous layer of space. The functionality of storing and organising frequently used objects could also be transferred from a classical screen-based desktop to a three-dimensional interface which occupies the space around the user's body. Further research based on this study may already be conducted in relation to a concrete application in the design of humancomputer interfaces.

There are limitations to the design of interfaces that occupy a continuous space, however. In the interfaces we interact with every day, we encounter a vast number of objects all of which we can potentially interact with. A logic of consecutive windows supports the interaction with large numbers of objects whereas a continuous space that needs to accommodate all objects comes to its boundaries as the number of objects is increased.

Thus, a selection needs to be made as to which objects a continuous space of interaction should contain. Such a selection could be enabled through configurability of the interface, or through automatic adaptation. The number of objects a space can contain while still being intelligible is likely to depend on its size and also on the patterns by which the objects are spatially organised. 


\section{Acknowledgments}

We would like to thank Nadia Berthouze, Sam Griffiths, Sean Hanna and Martha Tsigkari for the feedback and support in the course of this study.

\section{References}

1. Beck, A., Janssen, C., Weisbecker, A., Ziegler, J.: Integrating Object-Oriented Analysis and Graphical User Interface Design. Fraunhofer-Institut für Arbeitswirtschaft und Organisation (1993)

2. Allen, J., Chudley, J.: Smashing UX Design. Chichester: John Wiley \& Sons (2012)

3. John, B.E., Kieras, D.E.: Using GOMS for User Interface Design and Evaluation: Which Technique? ACM Transactions on Computer-Human Interaction, 3(4), 287-319 (1996)

4. Hornecker, E., Buur, J.: Getting a grip on Tangible Interaction: A Framework on Physical Space and Social Interaction. CHI 2006, April 22-28, 2006, Montreal, Québec, Canada (2006)

5. Ullmer, B., Ishii, H.: Emerging Frameworks for tangible user interfaces. IBM Systems Journal 39(3-4), 915-931 (2000)

6. Burgess, C.: From simple associations to the building blocks of language. Behavior Research Methods. Instruments \& Computers, 30(2), 188-198 (1998)

7. Lund, K., Burgess, C.: Producing high-dimensional semantic spaces from lexical cooccurrence. Behavior Research Methods. Instruments \& Computers, 28(2), 203-208 (1996)

8. Kalff, C., Strube, G.: Everyday navigation in Real and Virtual Environments Informed by Semantic Knowledge. In L. Carlson, C. Hoelscher, \& T.F. Shipley (Eds.), Proceedings of the 33rd Annual Conference of the Cognitive Science Society. Austin, TX: Cognitive Science Society (2011)

9. Fatah gen. Schieck, A., Moutinhou, A.: ArCHI - Engaging with museum objects spatially through whole body movement. In Academic MindTrek '12: International Conference on Media of the Future, Tampere, Finland (2012)

10. Sharlin, E., Watson, B., Kitamura, Y., Kishino, F., Itoh, Y.: On tangible user interfaces, humans and spatiality. Personal and Ubiquitous Computing 8(5), 338-346 (2004)

11. Robbins, P. , Aydede, M.: The Cambridge Handbook of Situated Cognition. Cambridge: Cambridge University Press (2009)

12. Laban, R.: Choreutics. Norwich: Jarrold and Sons Limited (1966)

13. Tversky, B., Morrison, J.B., Franklin, N., Bryant, D.J.: Three Spaces of Spatial Cognition. Professional Geographer, 51(4), 516-524 (1999)

14. Vingerhoets, G., Vandamme, K., Vercammen, A.: Conceptual and physical object qualities contribute differently to motor affordances. Brain and Cognition 69, 481-489 (2008)

15. Beach, K.: Becoming a Bartender: The Role of External Memory Cues in a Work-directed Educational Activity. Applied Cognitive Psychology, 7, 191-204 (1993)

16. Fatah gen. Schieck, A.: Embodied, mediated and performative: Exploring the architectural education in the digital age. In Voyatzaki, M., Spiridonidis, C.: Rethinking the human in technology-driven architecture. Transactions on Architectural Education No 55 (2012)

17. Gibson, J.J.: The ecological approach to visual perception. New York: Psychology Press (1986)

18. Moggridge, B.: Designing Interactions. Cambridge, Massachusetts: The MIT Press (2006)

19. Norman, D.A.: Affordance, Conventions, and Design. Interactions, may and june (1999) 
20. Dourish, P.: Where the Action is. Cambridge, Massachusetts: The MIT Press (2001)

21. Hardiess, G., Gillner, S., Mallot, A.: Head and eye movements and the role of memory limitations in a visual search paradigm. Journal of Vision, 8(1):7, 1-13 (2008)

22. Hardiess, G, Basten, K., Mallot, H.A.: Acquisition vs. Memorization Trade-Offs Are Modulated by Walking Distance and Pattern Complexity in a Large-Scale Copying Paradigm. PLoS ONE, 6(4) (2011)

23. Hillier, B.: The Social Logic of Space. New York: Cambridge University Press (1984)

24. Derry, S.J.: Cognitive Schema Theory in the Constructivist Debate. Educational Psychologist, 31(3/4), 163-174 (1996)

25. Hirtle, S.C., Jonides, J.: Evidence of hierarchies in cognitive maps. Memory \& Cognition, 13(3), 208-217 (1985)

26. Jameson, A.: Adaptive Interfaces and Agents. DFKI, German Research Center for Artificial Intelligence (2008)

27. Kuhn, G.: Die "Frankfurter Küche". Bonn: Wohnkultur und kommunale Wohnungspolitik in Frankfurt am Main. 1880-1930, 142-176 (1998)

28. Penn, A.: Space Syntax and Spatial Cognition. Environment and Behaviour, 35(1), 30-65 (2003)

29. Pirolli, P., Card, S. K.: Information foraging. Psychological Review, 106, 643-675 (1999)

30. Tversky, B.: Cognitive Maps, Cognitive Collages, and Spatial Mental Models. Lecture Notes in Computer Science, 716, 14-24 (1993)

31. Zacks, J.M., Tversky, B.: Event Structure in Perception and Conception. Psychological Bulletin 2001, Vol. 127, No.1, 3-21 (2001) 